Check for updates

Cite this: RSC Adv., 2018, 8, 6083

\title{
A novel fluorescent functional monomer as the recognition element in core-shell imprinted sensors responding to concentration of 2,4,6- trichlorophenol $\dagger$
}

\author{
Baixiang Ren, Huan Qi, Xiuying Li, Lihui Liu, Lin Gao, (D)* Guangbo Che, (DD* Bo Hu, \\ Liang Wang and Xue Lin
}

\begin{abstract}
We have demonstrated a fluorescent functional monomer instead of the traditional functional monomers for molecularly imprinted sensors. The sensors were firstly used to selectively detect 2,4,6-trichlorophenol $(2,4,6-\mathrm{TCP})$ by solid fluorescence detection without a dispersion solution. Moreover, the selectivity and anti-interference ability of the $\mathrm{SiO}_{2}$ adye-FMIPs sensor meet the requirements of a fluorescent sensor. The novel fluorescent monomer introduced into MIP is no longer just a fluorophore without recognizing ability. The fluorescence intensity of $\mathrm{SiO}_{2}$ adye-FMIPs showed a linear response to 2,4,6-TCP concentration in the range of 0-100 nM with a detection limit of $0.0534 \mathrm{nM}$. We could also demonstrate that such a system can not only get rid of the confines of traditional functional monomers and detection manner, but also improved the applications of MIPs sensors in sensing systems.
\end{abstract}

Received 14th July 2017

Accepted 23rd January 2018

DOI: $10.1039 / \mathrm{c} 7 \mathrm{ra0} 7742 \mathrm{~d}$

rsc.li/rsc-advances
The central part of a chemical sensor is the recognition element in order to assess the binding events directly with a sensitive analytical procedure, for example fluorescence detection technique. ${ }^{\mathbf{1 0}-15}$ Fluorescence detection based on fluorescent sensors is the most valuable method utilized in sensing thanks to its high sensitivity and simplicity. Fluorescence detection is the superior detection method in the field of sensing technology owing to several well-established advantages. Such signaling MIPs would be a sensor material and would expand the application of MIPs in fluorometric analysis instead of fluorescence label and displacement assays. ${ }^{16-21}$ MIPs are usually used to separate analytes as a selective sorbent. A further analysis will then be carried out by other apparatus, which is not ideal for sensor applications.

MIPs in which fluorescent moieties are directly incorporated in the polymer are scarce. Moreover, the covalently embedded dye can only be employed as a fluorophore without recognition ability. ${ }^{22,23}$ Perhaps the most feasible method, the covalent integration of a fluorescent probe monomer into an MIP, cannot however form the recognition sites with a functional monomer like methacrylic acid. ${ }^{24-27}$ As the most appealing type, if we can find a fluorescent functional monomer that catches an analyte by hydrogen bonds, hydrophobic interactions, and/or $\pi-\pi$ stacking interactions, the application of MIPs as a fluorescence sensor would provide satisfactory fluorometric analysis. To develop MIPs that show a reduction of fluorescence upon analyte binding and perform well in molecular recognition, we chose 7-allyloxycoumarin as the fluorescent functional monomer. It is constructed from a coumarin fluorophore and an
Key Laboratory of Preparation and Applications of Environmental Friendly Materials, Jilin Normal University, Ministry of Education, Changchun, 130103, People's Republic of China. E-mail: gaolinujs@163.com; guangboche@jlnu.edu.cn; Tel: +86 18843410256; +86 17704348899

$\dagger$ Electronic supplementary information (ESI) available. See DOI: $10.1039 / \mathrm{c} 7 \mathrm{ra07742d}$ 
allyloxy moiety, which can recognize templates via hydrogen bonds and $\pi-\pi$ interactions. In addition, most fluorogenic MIPs are simply dispersed in solution at the presence of analytes in an analytical process. So, the unsteady dispersion solution may bring about an inaccurate result. Maybe solid fluorescence detection can solve the problem.

2,4,6-Trichlorophenol (2,4,6-TCP), widely employed in the manufacturing of fungicides, herbicides, pesticides, insecticides, antiseptics, pharmaceuticals, dyes and plastics, has been listed as a priority pollutant by the US Environmental Protection Agency and the European Union, ${ }^{28}$ together with some other chlorophenol congeners. Adverse effects on human health caused by TCP, such as respiratory effects from coughs to serious pulmonary defects, gastrointestinal effects, and cardiovascular effects, have been reported. ${ }^{26}$ Even low levels of 2,4,6-TCP in drinking water may be a serious threat to human health and natural ecosystems. Therefore, it is important to monitor the concentration of 2,4,6-TCP for human health, safety and environmental protection purposes.

In this work, we chose 2,4,6-TCP as our template, 7-allyloxycoumarin as the fluorescent functional monomer, ethylene glycol dimethacrylate (EGDMA) as the crosslinker, and 3(methacryloxyl)propyltrimethoxysilane (MPS)-modified $\mathrm{SiO}_{2}$ spheres as the solid carrier to prepare an ideal fluorescent MIP sensor $\mathrm{SiO}_{2} @$ dye-MIPs.

\section{Experimental section}

\section{Materials and instruments}

Tetraethyl orthosilicate (TEOS), 3-(methacryloxyl)propyltrimethoxysilane (MPS), 2,4-dichlorophenol (2,4-DCP), 2,5dichlorophenol (2,5-DCP), 2,6-dichlorophenol (2,6-DCP), 2,4,6trichlorophenol (TCP), $N, N$-dimethylformamide (DMF, 99.5\%), allyl bromide, 7-hydroxycoumarin, ethylene glycol dimethacrylate (EGDMA), and 2,2'-azobis(2-methylpropionitrile) (AIBN) were obtained from Aladdin Reagent Co., Ltd. (Shanghai, China). Allyl bromide, acetonitrile, acetone, methanol, acetic acid and ammonia solution were all purchased from Sinopharm Chemical Reagent Co., Ltd. (Shanghai, China). Double distilled water was prepared in our laboratory and used for cleaning processes. All other chemicals used were of analytical grade and were obtained commercially.

${ }^{1} \mathrm{H}-\mathrm{NMR}$ spectroscopy was recorded on a Bruker AVANCE III HD400 NMR spectrometer. The morphologies of the samples were observed by an S-5500 scanning probe microscope and a transmission electron microscope (TEM, JEM-2100). Fluorescence intensity was measured using an F-4600 FL spectrophotometer.

\section{Synthesis of 7-allyloxycoumarin}

7-Allyloxycoumarin was prepared by Williamson reaction. A mixture of 7-hydroxycoumarin (1.62 g, $10.0 \mathrm{mmol})$, allyl bromide (2.42 g, $20.0 \mathrm{mmol}), \mathrm{K}_{2} \mathrm{CO}_{3}(4.97 \mathrm{~g}, 36.0 \mathrm{mmol})$ and acetone $(60 \mathrm{~mL})$ was heated and stirred at $57{ }^{\circ} \mathrm{C}$ in the dark under $\mathrm{N}_{2}$ for $10 \mathrm{~h}$. The solvent was evaporated under reduced pressure. The crude product was recrystallized from chloroform, and the resulting product was separated by column chromatography on silica to obtain the product as a white powder. ${ }^{1} \mathrm{H}$ NMR (400 MHz, $\left.\mathrm{CDCl}_{3}\right) \delta 7.63(\mathrm{~d}, J=9.5 \mathrm{~Hz}, 1 \mathrm{H})$, 7.37 (d, $J=8.6 \mathrm{~Hz}, 1 \mathrm{H}), 6.92-6.77(\mathrm{~m}, 2 \mathrm{H}), 6.25$ (d, $J=9.5 \mathrm{~Hz}$, $1 \mathrm{H}$ ), 6.04 (ddt, $J=17.2,10.6,5.3 \mathrm{~Hz}, 1 \mathrm{H}$ ), 5.39 (ddq, $J=36.3$, 10.5, $1.4 \mathrm{~Hz}, 2 \mathrm{H}), 4.60$ (dt, $J=5.3,1.5 \mathrm{~Hz}, 2 \mathrm{H}$ ), in Fig. S1.†

\section{Preparation of $\mathrm{SiO}_{2}$ beads modified by MPS}

$2 \mathrm{~mL}$ of ammonium hydroxide and $25 \mathrm{~mL}$ of double distilled water were added to $25 \mathrm{~mL}$ of ethanol and stirred for $15 \mathrm{~min}$. The mixture was poured into a $100 \mathrm{~mL}$ flask. Then, $2.0 \mathrm{~mL}$ of TEOS was added into the flask sequentially and stirred at $300 \mathrm{rpm}$ continuously at room temperature. After 6 hours, $\mathrm{SiO}_{2}$ spheres were separated by centrifuge and washed by ethanol and double distilled water.

The $\mathrm{SiO}_{2}$ spheres were redispersed in $40 \mathrm{~mL}$ of ethanol inside a round-bottomed flask and $1 \mathrm{~mL}$ of MPS was added into the flask. The round-bottomed flask was submerged in a thermostatically controlled oil bath at $40{ }^{\circ} \mathrm{C}$ and stirred at $300 \mathrm{rpm}$ for 24 hours. The resulting $\mathrm{SiO}_{2}-$ MPS spheres were separated by centrifuge from the solvent and washed five times sequentially in ultrasonic baths containing ethanol. Finally, the $\mathrm{SiO}_{2}-\mathrm{MPS}$ beads obtained were dried under vacuum for 12 hours at $40{ }^{\circ} \mathrm{C}$.

\section{Preparation of $\mathrm{SiO}_{2} @ d y e-F M I P$ and $\mathrm{SiO}_{2} @ d y e-F N I P$}

$\mathrm{SiO}_{2} @$ dye-FMIP and $\mathrm{SiO}_{2} @$ dye-FNIP were prepared by surface molecular imprinting technique (SMIT). $1.0 \mathrm{~g}$ of MPS-modified $\mathrm{SiO}_{2}$ beads was added to a $100 \mathrm{~mL}$ round-bottomed flask and dispersed in $60 \mathrm{~mL}$ of acetonitrile by sonication for $30 \mathrm{~min}$. Then, 2,4,6-TCP (0.394 g, $2.0 \mathrm{mmol})$, 7-allyloxycoumarin $(0.404 \mathrm{~g}, 2.0 \mathrm{mmol})$ and EGDMA $(1.88 \mathrm{~mL}, 10.0 \mathrm{mmol})$ were dissolved in the round-bottomed flask in a glove box with $\mathrm{N}_{2}$. The reaction system was sparged with oxygen-free nitrogen for 15 min to expel the oxygen present inside the reaction flask. The flask was then submerged in a thermostatically controlled oil bath at $70{ }^{\circ} \mathrm{C}$. After 3 hours, the $\mathrm{SiO}_{2} @$ dye-FMIPs particles were collected from the reaction medium by centrifuge and then cleaned successively with methanol/acetic acid $(100 \mathrm{~mL}, 95 / 5 \mathrm{v} /$ v) to remove the templates by Soxhlet extractor. Finally, the product was dried in vacuo overnight at $40{ }^{\circ} \mathrm{C}$. Fluorescent $\mathrm{SiO}_{2}$ @dye-NIPs were prepared under nominally duplicate conditions to those used for the $\mathrm{SiO}_{2}$ @dye-FMIPs in the absence of the 2,4,6-TCP template. By gravimetric analysis, the yields of $\mathrm{SiO}_{2} @$ @ye-FMIPs and $\mathrm{SiO}_{2}$ @dye-FNIPs were found to be $87 \%$ and $83 \%$, respectively.

\section{The solid fluorescence detection experiments}

The samples of $\mathrm{SiO}_{2}$ @dye-FMIPs (100.0 mg) were dispersed in $100.0 \mathrm{~mL}$ of alcohol solution for spectrum measurement. After that, 2,4,6-TCP solutions in ethanol were prepared at various concentrations in the range of 0-1000 nmol. For the fluorescent measurements, $5 \mathrm{~mL}$ of the sample solution was mixed with $5 \mathrm{~mL}$ of the 2,4,6-TCP solutions of different concentrations. After 1.0 hour of dipping, the solids were separated by centrifuge and dried in vacuo overnight at $40{ }^{\circ} \mathrm{C}$. Then a fluorescence spectrophotometer was used to detect the fluorescence intensity 
of the $\mathrm{SiO}_{2} @ d y e-F M I P s$ sensors. Fluorescence spectra were measured under a $332 \mathrm{~nm}$ excitation light source. The fluorescence quenching efficiency of the $\mathrm{SiO}_{2}$ @dye-FMIPs sensors with 2,4,6-TCP was calculated by Stern-Volmer equation $\left(I_{0} / I\right)-1=$ $K_{\mathrm{SV}} C\left(I_{0}\right.$ is the initial fluorescence intensity without analyte, $I$ is the fluorescence intensity of the $\mathrm{SiO}_{2} @$ adye-FMIPs with different standard 2,4,6-TCP concentrations, and $K_{\mathrm{SV}}$ is the quenching constant with 2,4,6-TCP).

\section{Selectivity experiments}

To estimate the selectivity of the $\mathrm{SiO}_{2}$ @dye-FMIPs sensor, we made a comparison between three structurally related compounds and 2,4,6-TCP. The $100 \mathrm{mg} \mathrm{S}$ (adye-FMIP sensors were added to $100 \mathrm{~mL}$ of ethanol solutions containing 20.0 nmol of 2,4,6-TCP, 2,4-TCP, 2,5-TCP or 2,6-TCP. The mixtures were stirred for 1 hour at room temperature and solids were separated by centrifuge. The fluorescence intensity of the solids was detected by fluorescence spectrophotometer and the $\left[\left(I_{0} / I\right)\right.$ -1 ] was calculated with the fluorescence data. For comparison, the same procedure was applied using $\mathrm{SiO}_{2} @$ @ye-FNIPs instead of $\mathrm{SiO}_{2} @$ dye-FMIPs.

\section{Interference experiments}

The capacity to resist interferents is one key fact to evaluate whether a sensor would be used in practical samples. To evaluate the $\mathrm{SiO}_{2}$ @dye-FMIPs sensor's capacity of resisting interferents, $100 \mathrm{mg}$ of $\mathrm{SiO}_{2}$ @dye-FMIP was added into $100 \mathrm{~mL}$ of ethanol solutions containing $20.0 \mathrm{nmol}$ of 2,4,6-TCP, 2,4-TCP, 2,5-TCP or 2,6-TCP. After stirring for 1 hour at room temperature, solids were separated by centrifuge. The fluorescence intensity of the solids was detected by fluorescence spectrophotometer.

\section{Results and discussion}

One of the factors that limits the application of fluorescent molecular imprinting is the complexity of the fluorescent functional monomer. Herein, we adopted the Williamson reaction to synthesize 7-allyloxycoumarin, which greatly simplified the process of the preparation of fluorescent functional monomers. As-synthesized 7-allyloxycoumarin not only had a recognition group but could also polymerize with other monomers (Scheme 1). To our knowledge, there are no reports about 7-allyloxycoumarin being used for MIPs, probes or sensors. The crucial interaction between 7-allyloxycoumarin and 2,4,6-TCP is hydrogen bonding because the absorption peak position of 2,4,6-TCP has a blue shift of $12 \mathrm{~nm}$ after adding 7-allyloxycoumarin in dichloromethane (in Fig. S2†). This blue shift in the absorption spectra might be attributed to the hydrogen bond between 2,4,6-TCP and 7-allyloxycoumarin. Versus covalent methods, non-covalent formation of MIPs is most often employed for introducing functionality into MIPs because of easier methodology and faster elution as a consequence of the rapid and reversible nature of the non-covalent interaction between the polymer and the template.

As an alternative to molecular imprinting technique, surface molecular imprinting technique has emerged as an attractive, simple, and seemingly general method for facilitating the production process of imprinted products. The reason that we chose $\mathrm{SiO}_{2}$ microspheres as the solid support is because they possess a vast surface area, physical robustness and thermal stability, and can also be integrated in MIPs membranes. ${ }^{29,30}$ Silica particles of about $255 \mathrm{~nm}$ were used as the support, as measured from the SEM image (Fig. S3 $\dagger$ ). The pure $\mathrm{SiO}_{2}$ @dyeFMIPs was highly spherical and monodisperse with an average size of about $275 \mathrm{~nm}$, as shown in Fig. S4. $\dagger$ A representative TEM image of the pure $\mathrm{SiO}_{2} @ d y e-F M I P s$ is shown in Fig. S5; $\uparrow$ when MPS-modified $\mathrm{SiO}_{2}$ spheres were coupled with the dye-FMIP layer, the grain size was increased by about $20 \mathrm{~nm}$. In order to make 7-allyloxycoumarin copolymerise with other monomers in the MIP system, allyl groups were attached to the hydroxyl by reacting with allyl bromide. The structural properties of the synthesised $\mathrm{SiO}_{2} @$ adye-FMIPs microspheres were analysed by FT-IR spectra. As shown in Fig. S6, $\uparrow$ the characteristic peaks at 1730,1258 and $1151 \mathrm{~cm}^{-1}$ are attributed to the stretching of the $\mathrm{C}=\mathrm{O}$ and $\mathrm{C}-\mathrm{O}$ of EGDMA. The typical peaks of 2986 and $2956 \mathrm{~cm}^{-1}$ proved that 7-allyloxycoumarin had copolymerised with other monomers in the MIP system. The strong and broad peak around $1103 \mathrm{~cm}^{-1}$ indicated the $\mathrm{Si}-\mathrm{O}-\mathrm{Si}$ asymmetric stretching. These FT-IR spectra suggest that the $\mathrm{SiO}_{2} @$ @dye-FMIPs have been successfully prepared.

The performances of the $\mathrm{SiO}_{2} @$ @dye-FMIPs and $\mathrm{SiO}_{2} @$ dyeFNIPs sensors were assessed by solid fluorescence with 2,4,6TCP. Structural analogues 2,4-dichlorophenol (2,4-DCP), 2,5dichlorophenol (2,5-DCP), and 2,6-dichlorophenol (2,6-DCP) were chosen as potential competitors. Solid fluorescence spectra were measured under a $332 \mathrm{~nm}$ excitation light source at room temperature. To study the fluorescence quenching mechanism of $\mathrm{SiO}_{2}$ @dye-FMIPs sensors with 2,4,6-TCP, the quenching efficiency of $\mathrm{SiO}_{2}$ @dye-FMIPs was evaluated by the Stern-Volmer equation $\left(I_{0} / I\right)-1=K_{\mathrm{SV}} C\left(I_{0}\right.$ is the initial fluorescence intensity of $\mathrm{SiO}_{2}$ @dye-FMIPs, $I$ is the peak of the $\mathrm{SiO}_{2}$ @dye-MIPs with different standard 2,4,6-TCP, and $K_{\mathrm{Sv}}$ is the quenching constant with 2,4,6-TCP).

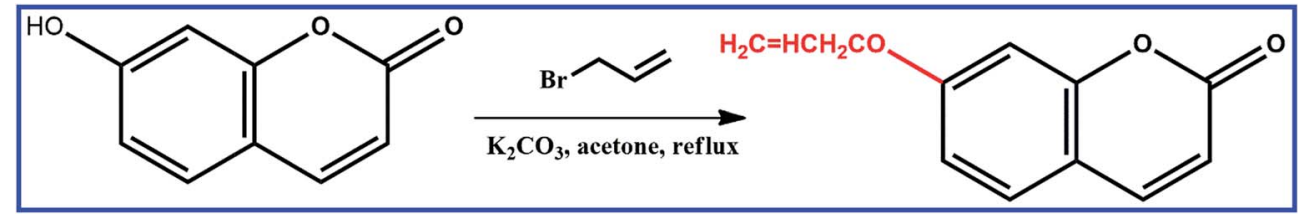

Scheme 1 Schematic illustration of the synthesis of 7-allyloxycoumarin. 
As shown in Fig. 1, a significant inverse relation was found between fluorescence intensity and 2,4,6-TCP concentration. The more 2,4,6-TCP that is added, the weaker the fluorescence intensity is. It means that the fluorescence intensity of $\mathrm{SiO}_{2}$ @dye-FMIPs (Fig. 1a) decreases with increasing 2,4,6-TCP concentration, and the reduced degree of fluorescent $\mathrm{SiO}_{2} @$ @dye-FMIPs was notably higher than that of the fluorescent $\mathrm{SiO}_{2}$ @dye-FNIPs (Fig. 1b). It is illustrated that the spatial adsorption sites could be incorporated into the $\mathrm{SiO}_{2}$ @dye-FMIPs matrix, but not in $\mathrm{SiO}_{2}$ @dye-FNIPs. Therefore, it is also confirmed that $\mathrm{SiO}_{2}$ @dye-FMIPs particles are more sensitive than $\mathrm{SiO}_{2} @$ dye-FNIPs particles.

The excellent linearity of the technique was investigated though the relationship of the fluorescence intensity with the concentration of 2,4,6-TCP in a working range from 0 to $100 \mathrm{nM}$, as shown in Fig. 2. The linear equation of the $\mathrm{SiO}_{2}$ @dye-FMIPs sensor was $\left(I_{0} / I\right)-1=0.01051 C_{\mathrm{c}}+0.00582$ (where $C_{\mathrm{c}}$ is the concentration of $2,4,6-\mathrm{TCP}$ in $\mathrm{nM}$, and $\left(I_{0} / I\right)-1$ is the relative fluorescence intensity), and the corresponding correlation coefficient $\left(R^{2}\right)$ was 0.99947 . The limit of detection was evaluated using $3 s / S$, and was found to be $0.0534 \mathrm{nM}$ ( $s$ is the standard deviation of the blank signal and $S$ is the slope of the linear calibration plot). From the figure, we can see that the linear concentration range of $\mathrm{SiO}_{2}$ @dye-FMIPs is much larger than that of $\mathrm{SiO}_{2}$ @dye-FNIPs. This is because the quenching only took place between 7-allyloxycoumarin and 2,4,6-TCP at the surface of the $\mathrm{SiO}_{2}$ @dye-FNIPs particles with no recognition sites, and did not happen both inside and outside like with the $\mathrm{SiO}_{2}$ @dye-FMIPs particles.

It is well known that fluorescence quenching processes includes dynamic quenching and static quenching. In a dynamic quenching process, excited state molecules and quencher molecules collide with each other, leading to transition of the excited state molecule back to the ground state. At the same time, the lifetime of the fluorescent material reduces with the variety of fluorescence intensity. Static quenching happens between the quenching agent and the fluorescent molecule in the ground state, but the fluorescence lifetime is not changed. Both dynamic quenching and static quenching processes can be described by the Stern-Volmer equation:

$$
I_{0} / I=1+K_{\mathrm{SV}}[C]=1+K_{\mathrm{q}} \tau_{0}[C]
$$

$I_{0}$ is the initial fluorescence intensity without analyte. $I$ is the fluorescence intensity in the presence of analyte. $K_{\mathrm{SV}}$ is the
Stern-Volmer quenching constant in units of $\mathrm{L} \mathrm{mol}^{-1} . C$ is the concentration of the molecular target. $K_{\mathrm{q}}$ is the rate constant of the bimolecular quenching process in units of $\mathrm{L} \mathrm{mol}^{-1} \mathrm{~s}^{-1} \cdot \tau_{0}$ is the lifetime without quenching agent.

Time-resolved fluorescence curves of $\mathrm{SiO}_{2} @$ @dye-FMIPs before and after adsorbing 2,4,6-TCP are shown in Fig. 3. The two decay curves were fitted by exponential function. The nonlinear equations of $\mathrm{SiO}_{2} @$ adye-FMIPs before and after adsorbing 2,4,6-TCP are $I(t)=42065.8 \exp (-t / 4.01076)+$ 0.00304 and $I(t)=2.47042 \times 10^{10} \exp (-t / 1.79746)+0.00363$, and the corresponding correlation coefficients $\left(R^{2}\right)$ were $R^{2}=$ 0.98147 and $R^{2}=0.98801$, respectively. From the equations, we found that the lifetimes were $\tau_{0}=4.01076 \mathrm{~ns}$ and $\tau=1.79746$ $\mathrm{ns}$, respectively. The lifetimes were quite different in the presence and absence of 2,4,6-TCP. We found another kind of representation of the Stern-Volmer equation according to eqn (1):

$$
\tau_{0} / \tau=1+K_{\mathrm{SV}} C=1+K_{\mathrm{q}} \tau_{0} C
$$

where $\tau$ is the lifetime with quenching agent.

According to eqn (2), we found the relationship $K_{\mathrm{SV}}=K_{\mathrm{q}} \tau_{0}$. $K_{\mathrm{SV}}$ is $0.01051 \mathrm{~L} \mathrm{~mol}^{-1}$, as shown in Fig. 2. Therefore $K_{\mathrm{q}}$ is $2.6 \times$ $10^{6} \mathrm{~L} \mathrm{~mol}^{-1} \mathrm{~s}^{-1}$. The rate constant $\left(K_{\mathrm{q}}\right)$ of maximum diffusion controlled dynamic quenching is $2.0 \times 10^{10}$. The $K_{\mathrm{q}}$ in here is always $<2.0 \times 10^{10} \mathrm{~L} \mathrm{~mol}^{-1} \mathrm{~s}^{-1}$. In addition, the lifetime of fluorescent sensors reduces with the variety of fluorescence intensity. Thus, it is confirmed that dynamic quenching is a dominant process in this work.

Selectivity is a very important index for a sensor. To evaluate the selective recognition property of the $\mathrm{SiO}_{2}$ @dye-FMIPs sensor, we chose structural analogues 2,4-DCP, 2,5-DCP, and 2,6-DCP as the competitors. Fig. 4 shows the fluorescence quenching efficiency of $\mathrm{SiO}_{2}$ @dye-FMIPs for 2,4,6-TCP, 2,4DCP, 2,5-DCP and 2,6-DCP. The fluorescence quenching efficiencies $\left(I_{0} / I\right)-1$ are $0.249,0.024,0.011$ and 0.02 , respectively. So, the fluorescence quenching efficiency of 2,4,6-TCP is much higher than other competitors. The results showed that none of the competitors being evaluated led to significant fluorescence quenching and the $\mathrm{SiO}_{2} @$ dye-FMIPs sensor had a specific affinitive action owing to its recognition sites. In order to evaluate the selectivity, an imprinted selectivity factor was calculated using the following eqn (3):

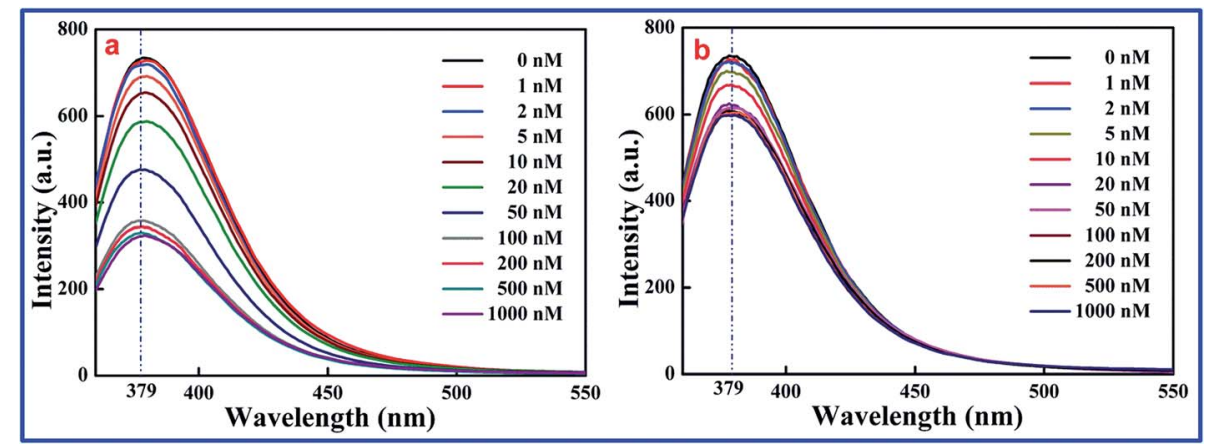

Fig. 1 Response of $\mathrm{SiO}_{2}$ (adye-MIPs (a) and $\mathrm{SiO}_{2}$ (adye-NIPs (b) to 2,4,6-TCP in the concentration range from 0 to $1000.0 \mathrm{nM}$. 

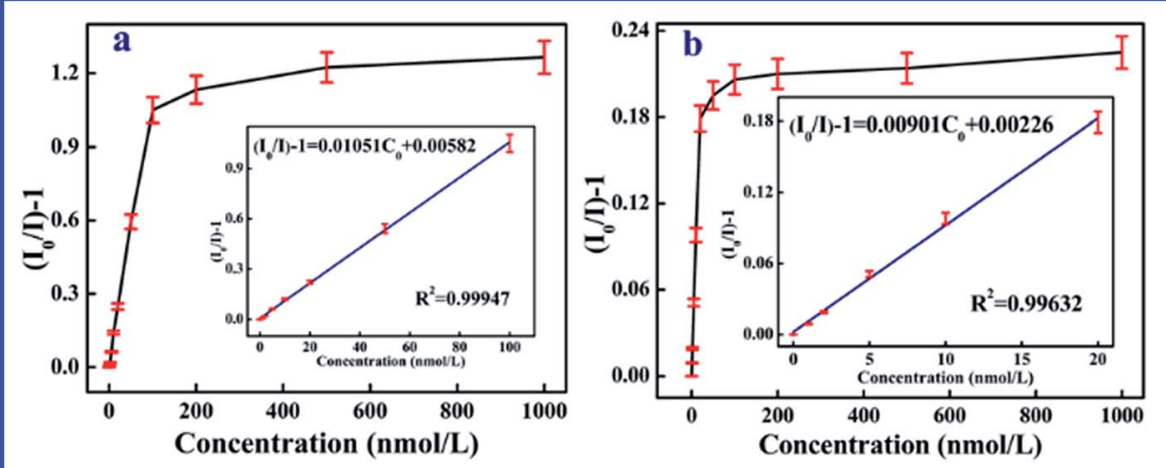

Fig. 2 Linear response of the $\mathrm{SiO}_{2}$ @ dye-MIPs (a) and $\mathrm{SiO}_{2}$ (adye-NIPs (b) to 2,4,6-TCP in the concentration range from 0 to $1000 \mathrm{nM}$.
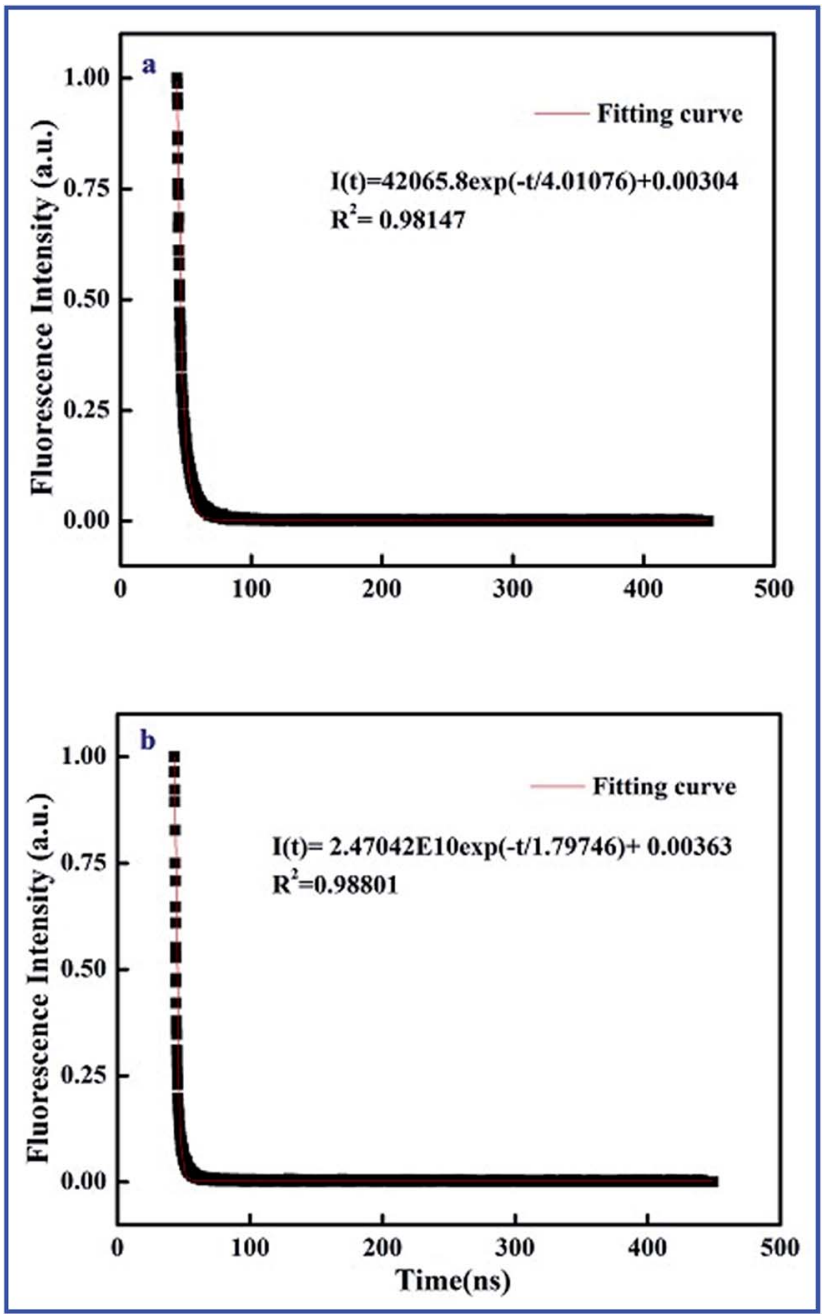

Fig. 3 Time-resolved fluorescence curves of $\mathrm{SiO}_{2} @$ adye-FMIPs before (a) and after (b) adsorbing 2,4,6-TCP

Imprinted selectivity factor $=\left(I_{\mathrm{MIP}}-I_{0}\right) /\left(I_{\mathrm{NIP}}-I_{0}\right)$

here, $I_{\mathrm{MIP}}$ is the fluorescence intensity of $\mathrm{SiO}_{2}$ @dye-FMIPs with $100 \mathrm{nM}$ 2,4,6-TCP, $I_{\mathrm{NIP}}$ is the fluorescence intensity of $\mathrm{SiO}_{2} @$ @dye-FNIPs with $100 \mathrm{nM} 2,4,6-\mathrm{TCP}$, and $I_{0}$ is the

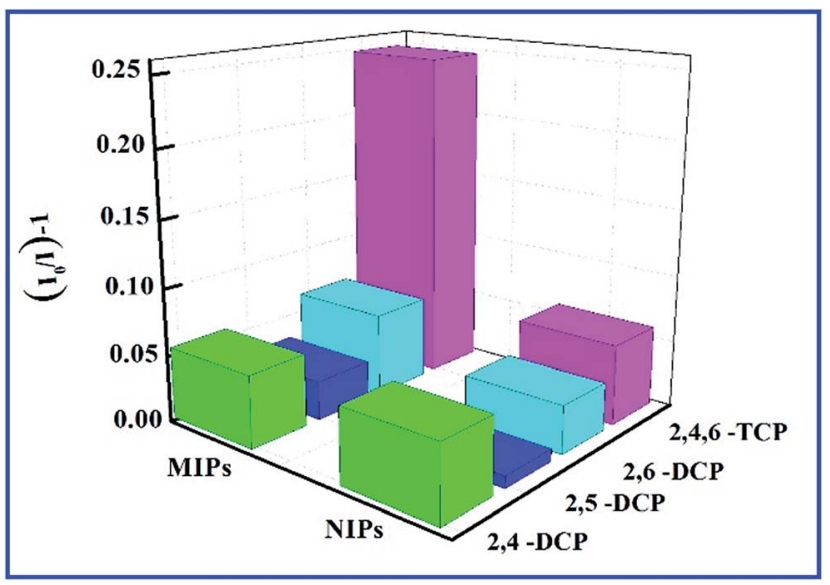

Fig. 4 Quenching amount of $\mathrm{SiO}_{2}$ adye-FMIPs and $\mathrm{SiO}_{2}$ (adye-FNIPs by different kinds of chlorophenols at $20 \mathrm{nmol} \mathrm{L}^{-1}$.

background fluorescence intensity without 2,4,6-TCP. If the imprinted selectivity factor is higher than 1.0 , it indicates that the MIPs exhibited good selectivity for 2,4,6-TCP. Finally, the imprinted selectivity factor obtained from fluorescence measurements was 2.95 , which proved that $\mathrm{SiO}_{2}$ @dye-FMIPs possessed high selectivity for 2,4,6-TCP.

To further investigate the interference of 2,4-DCP, 2,5-DCP, and 2,6-DCP, the three competitors were mixed with same concentration of 2,4,6-TCP to form a mixture. In Fig. 5, the fluorescence intensity of the $\mathrm{SiO}_{2}$ @dye-FMIPs sensor was changed unobviously for the three competitors, and the competitors being evaluated did not give any significant interference. This proves again that the $\mathrm{SiO}_{2}$ @dye-FMIPs sensor has high selectivity for 2,4,6-TCP. The higher selectivity for 2,4,6TCP results from its specific binding affinity of 2,4,6-TCP for an efficient imprinting effect because the same fluorescence quenching does not happen for $\mathrm{SiO}_{2}$ @dye-FNIPs particles.

In order to assess the practical efficiency of the $\mathrm{SiO}_{2} @$ @yeFMIPs sensor for the analysis of food samples, soda water purchased at Chinese supermarkets was used as a sample material. $5.0 \mathrm{~mL}$ of soda water was mixed with $5 \mathrm{~mL}$ of 2,4,6-TCP solution (concentration range $0-100 \mathrm{nM}$ ) and analyzed using the procedure discussed in Section 2. The results listed in Table 


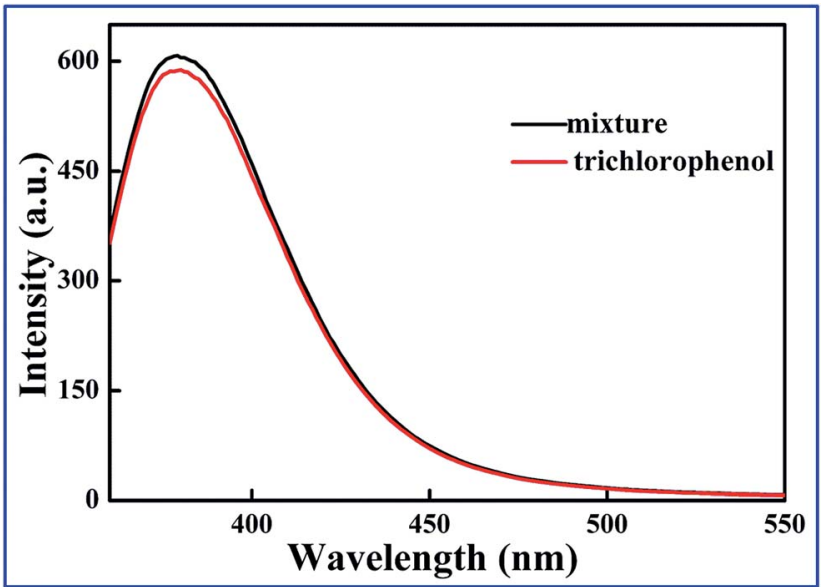

Fig. 5 Test for the interference of different chlorophenols on the fluorescence response toward 2,4,6-TCP of $\mathrm{SiO}_{2}$ @dye-FMIPs sensor.

Table 1 Recovery of 2,4,6-TCP in soda water samples at different concentration levels

\begin{tabular}{llll}
\hline Samples & $\begin{array}{l}\text { Concentration } \\
\text { taken }(\mathrm{nM})\end{array}$ & $\begin{array}{l}\text { Concentration } \\
\text { found }(\mathrm{nM})\end{array}$ & Recovery $(\%)$ \\
\hline \multirow{2}{*}{$2,4,6-\mathrm{TCP}$} & 0 & - & - \\
& 1 & 0.98 & 98 \\
& 2 & 2.19 & 108 \\
& 5 & 5.13 & 103 \\
10 & 10.22 & 102 \\
& 20 & 20.87 & 104 \\
& 50 & 49.13 & 98 \\
& 100 & 101.45 & 101 \\
& & & \\
\end{tabular}

1 clearly demonstrate that $\mathrm{SiO}_{2}$ @dye-MIPs sensor is capable of quantitatively measuring 2,4,6-TCP content in the concentration range of $0-100 \mathrm{nM}$. The results intelligibly established that $\mathrm{SiO}_{2}$ @dye-MIPs could attain good recovery and could be effectively applied in the detection of 2,4,6-TCP in soda water samples. Compared to gas chromatography (GC), ${ }^{31}$ the method in this work can selectively detect 2,4,6-TCP in complicated samples without interference from analogues. In addition, $\mathrm{SiO}_{2} @$ @dye-FMIPs could be used to monitor trace 2,4,6-TCP with a lower limit of detection $(0.0534 \mathrm{nM})$, while the limit of detection for the GC method is $0.25 \mu \mathrm{g} \mathrm{kg}{ }^{-1}$, which is much higher than the present method.

\section{Conclusions}

In conclusion, we have demonstrated a fluorescent functional monomer instead of a traditional functional monomer for SMIP sensors. The sensors were firstly used to selectively detect analyte by solid fluorescence detection without a dispersion solution. Moreover, the selectivity and anti-interference ability of the $\mathrm{SiO}_{2}$ @dye-MIPs sensor meet the requirements of a fluorescent sensor. The novel fluorescent monomer introduced into MIP is not only as a fluorophore but also as a functional monomer for recognizing the analyte. We could also demonstrate that such a system can not only get rid of the confines of the traditional functional monomers and detection manner, but also improved the applications of MIPs sensors in sensing systems.

\section{Conflicts of interest}

The authors declare no conflict of interest.

\section{Acknowledgements}

This work was financially supported by the National Natural Science Foundation of China (No. 21576112, 21407064, 21407057, 21407059, 201407056), the Innovation Foundation Project of Jilin Province (No. 20180623042TC), the Natural Science Foundation Project of Jilin Province (No. 20170520143JH and 20170520147JH), the China Postdoctoral Science Foundation (No. 2017M611732), and the Science and Technology Development Plan of Siping City (2017056 and 2014052).

\section{References}

1 M. J. Whitcombe and E. N. Vulfson, Adv. Mater., 2001, 13, 467-478.

2 B. T. S. Bui and K. Haupt, Anal. Bioanal. Chem., 2010, 398, 2481-2492.

3 L. Zhang, L. Chen, H. Zhang, Y. Z. Yang and X. G. Liu, J. Appl. Polym. Sci., 2017, DOI: 10.1002/app.45468.

4 R. N. Liang, D. A. Song, R. M. Zhang and W. Qin, Angew. Chem., Int. Ed., 2010, 49, 2556-2559.

5 S. Marx, A. Zaltsman, I. Turyan and D. Mandler, Anal. Chem., 2004, 76, 120-126.

6 S. A. Piletsky, E. V. Piletskaya, A. V. Elgersma, K. Yano and I. Karube, Biosens. Bioelectron., 1995, 10, 959-964.

7 L. Zhu, Y. Cao and G. Cao, Biosens. Bioelectron., 2014, 54, 258-261.

8 M. Goreti, F. Sales and L. Brandão, Biosens. Bioelectron., 2017, 98, 428-436.

9 W. Zhang, H. W. Xiong, M. M. Chen, X. H. Zhang and S. F. Wang, Biosens. Bioelectron., 2017, 96, 55-61.

10 O. Y. F. Henry, D. C. Cullen and S. A. Piletsky, Anal. Bioanal. Chem., 2005, 382, 947-956.

11 M. C. Moreno-Bondi, F. Navarro-Villoslada, E. Benito-Pena and J. L. Urraca, Curr. Anal. Chem., 2008, 4, 316-340.

12 A. Waggoner, Curr. Opin. Chem. Biol., 2006, 10, 62-66.

13 J. Yao, M. Yang and Y. X. Duan, Chem. Rev., 2014, 114, 61306178.

14 G. N. Wang and X. G. Su, Analyst, 2011, 136, 1783-1798.

15 M. Amjadi and R. Jalili, Biosens. Bioelectron., 2017, 96, 121126.

16 C. B. Liu, Z. L. Song, J. M. Pan, X. Wei, L. Gao, Y. S. Yan, L. Z. Li, J. Wang, R. Chen, J. D. Dai and P. Yu, J. Phys. Chem. C, 2013, 117(20), 10445-10453.

17 Y. Li, C. K. Dong, J. Chu, J. Y. Qia and X. Li, Nanoscale, 2011, 3, 280-287. 
18 Y. Y. Zhao, Y. X. Ma, H. Li and L. Y. Wang, Anal. Chem., 2012, 84, 386-395.

19 L. Gao, J. X. Wang, X. Y. Li, Y. S. Yan, C. X. Li and J. M. Pan, Anal. Bioanal. Chem., 2014, 406, 7213-7220.

20 L. Gao, W. J. Han, X. Y. Li, J. X. Wang, Y. S. Yan, C. X. Li and J. D. Dai, Anal. Bioanal. Chem., 2015, 407, 9177-9184.

21 W. Wan, M. Biyikal, R. Wagner, B. Sellergren and K. Rurack, Angew. Chem., Int. Ed., 2013, 52, 7023-7027.

22 Y. Liao, W. Wang and B. H. Wang, Bioorg. Chem., 1999, 27, 463-476.

23 R. Y. Liu, G. J. Guan, S. H. Wang and Z. P. Zhang, Analyst, 2011, 136, 184-190.

24 J. M. Pan, B. wang, J. D. Dai, X. H. Dai, H. Hang, H. X. Ou and Y. S. Yan, J. Mater. Chem., 2012, 22(8), 3360-3369.

25 J. M. Pan, W. Hu, X. H. Dai, W. Guan, X. H Zou, X. Wang, P. W. Huo and Y. S. Yan, J. Mater. Chem., 2011, 21(39), 15741-15751.
26 J. M. Pan, H. Yao, L. C. Xu, H. X. Ou, P. W. Huo, X. X. Li and Y. S. Yan, J. Phys. Chem. C, 2011, 115(13), 5440-5449.

27 H. Zaghouane-Boudiaf, M. Boutahalaa, C. Tiar, L. Arab and F. Garin, Chem. Eng. J., 2011, 173, 36-41.

28 Epa, Ambient water quality for chlorinated phenols, US Environmental Protection Agency, 1980, Available from, http://www.epa.gov/ost/pc/ambientwqc/ chlorinatedphenols80.pdf.

29 W. J. Cheng, Z. J. Liu and Y. Wang, Talanta, 2013, 116, 396402.

30 J. Li, X. B. Zhang, Y. X. Liu, H. W. Tong, Y. P. Xu and S. M. Liu, Talanta, 2013, 117, 281-287.

31 M. Zhang, J. Cheng, M. Wu, T. Du, X. H. Wang and M. Cheng, Anal. Methods, 2014, 6, 207-214. 